\title{
Lecture commentée de « Notes sur le calendrier japonais » d'André Leroi-Gourhan
}

Jane Cobbi

\section{(2) OpenEdition}

1 Journals

Édition électronique

URL : https://journals.openedition.org/tc/5786

DOI : $10.4000 /$ tc. 5786

ISBN : 1952-420X

ISSN : 1952-420X

Éditeur

Éditions de l'EHESS

Édition imprimée

Date de publication : 15 décembre 2011

Pagination : $54-59$

ISBN : 978-2-7351-1437-5

ISSN : 0248-6016

\section{Référence électronique}

Jane Cobbi, «Lecture commentée de « Notes sur le calendrier japonais » d'André Leroi-Gourhan »,

Techniques \& Culture [En ligne], 57 | 2011, mis en ligne le 30 juin 2012, consulté le 29 septembre 2022.

URL : http://journals.openedition.org/tc/5786 ; DOI : https://doi.org/10.4000/tc.5786 


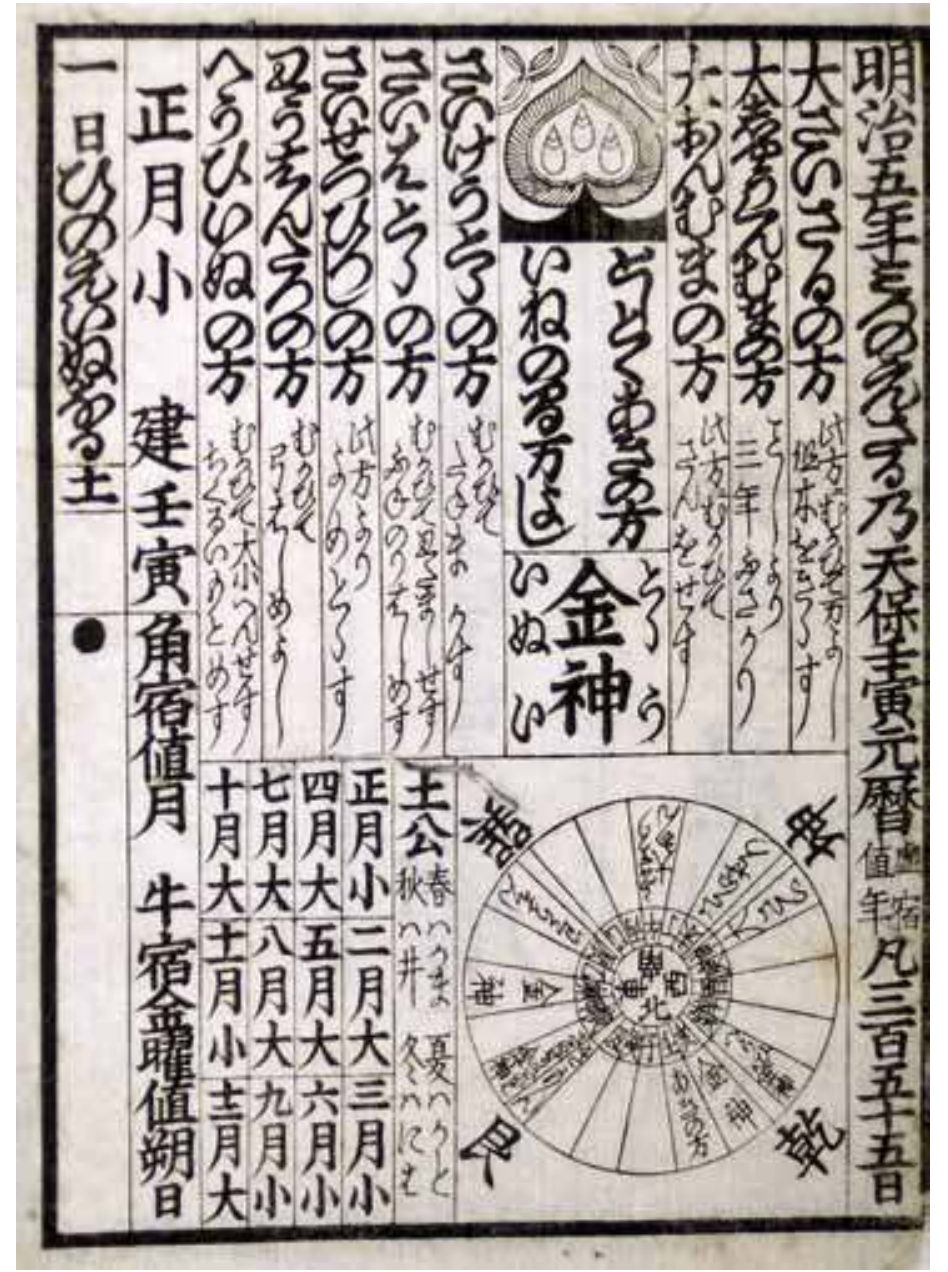

Lecture commentée de

\section{« Notes sur le calendrier japonais »}

Dans ce court texte, publié en 1947 sous le titre Calendrier des Fêtes populaires, André Leroi-Gourhan aborde les grandes lignes du calendrier japonais en mettant l'accent sur la succession des fêtes organisées dans les temples bouddhiques et les sanctuaires shinto. D'entrée, le lecteur est prévenu : il s'agit d'une « simple mise en ordre de notes de terrain ». Lénumération donnée correspond en effet aux cérémonies auxquelles il était (et est encore pour l'essentiel), le plus aisé d'assister, pour un étranger vivant à Kyoto. En annonçant d emblee la couleur, lauteur nous permet d imaginer un pan non traité dans ce exte et qui sera partiellement couvert par dautres analyses, conme letude impressionnante du vêtement japonais (Leroi-Gourhan 1946), ou les développements très amples qui jalonnent ses ouvrages, notamment Le Geste et la parole, ou les systemes calenderiques sont évoqués dans un chapitre consacré au temps (Leroi-Gourhan 1965 : 142-147).

Le Japon utilisait, à l'instar de la Chine, le calendrier luni-solaire, dans lequel on fixait le premier et le quinzième jour du mois d'après la nouvelle et la pleine lune, tout en suivant la course du soleil, et cela jusqu'à l'adoption du calendrier occidental, au xixe siècle, qui a remplacé le traditionnel calendrier luni-solaire, dans un souci de simplification. Le changement de calendrier a été décidé par décret, en 1872, plus précisément le ge jour du $1 l^{e}$ mois de l'année 5 de l'ère Meiji. En adoptant le calendrier solaire qui fixe les mois en suivant la course du soleil, "d'une très grande précision, et d'une commodité d'usage évidente r, les autoriés ont voulu fare dispars les subue sions traditionnelles de saisons, ainsi que les jours fastes et néfastes, infor subiqui qui « appartiennent aux croyances sans fondement et gênent le développement de la 

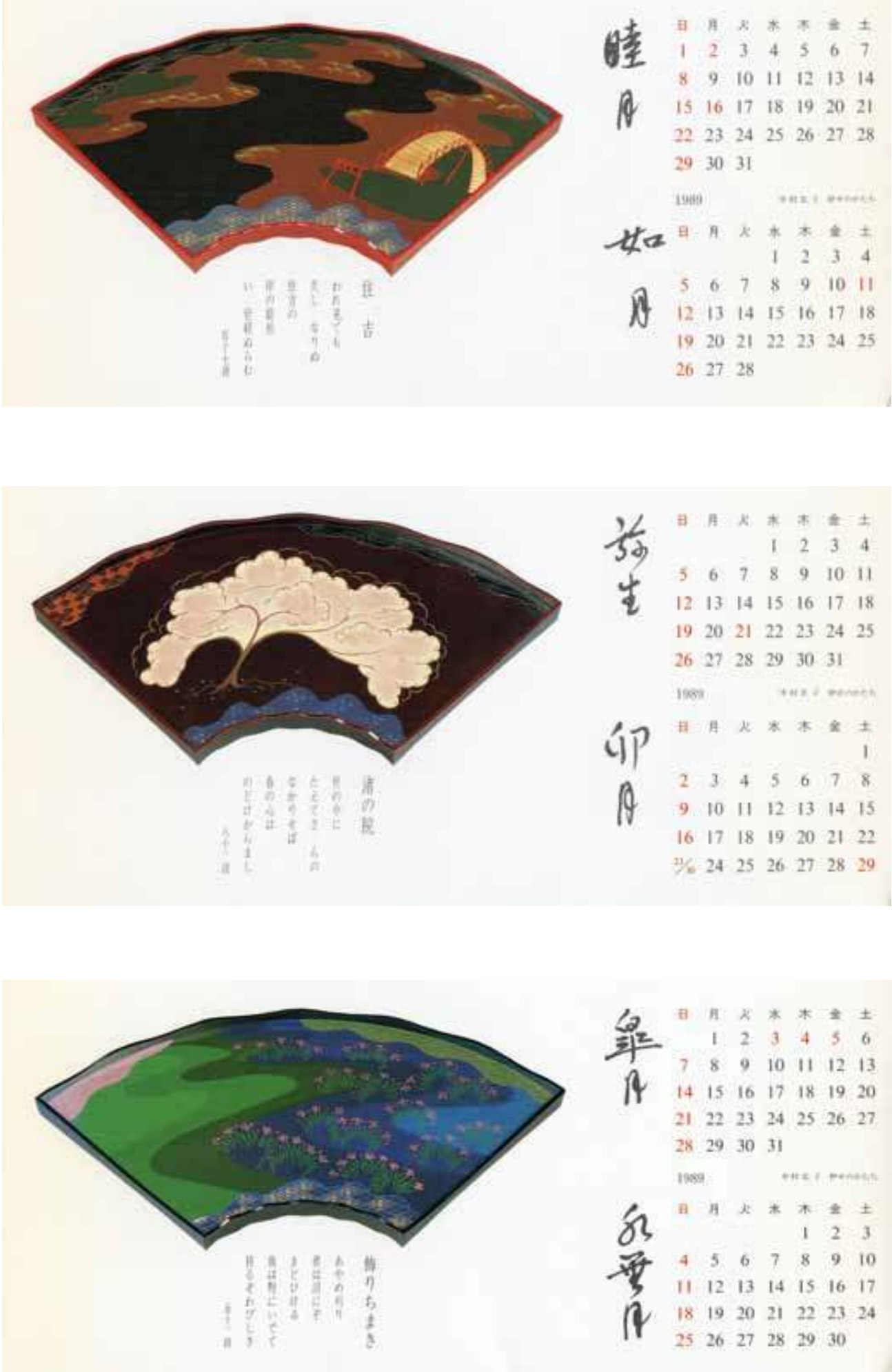
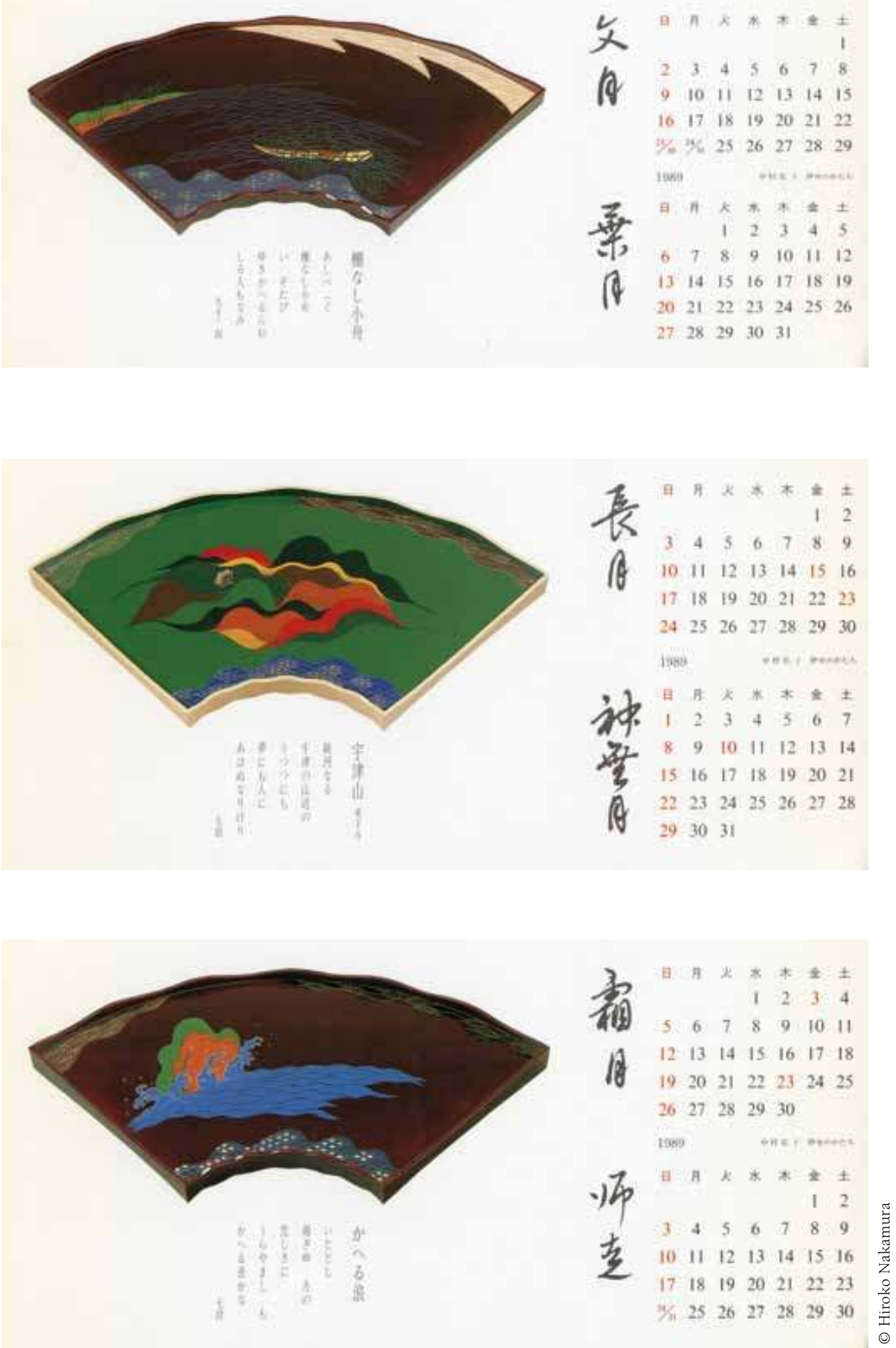
Ladministration et la plus grande partie des intellectuels ont adopté rapidement le nouveau calendrier mais, considérant que « changer de calendrier pour un pays est une affaire de la plus haute importance », l'écrivain Y. Fukuzawa jugea qu'il « est essentiel de donner a l'ensemble du peuple les raisons, d'expliquer sans relâche et avec soin les defférences entre les deux calendriers, de montrer leurs avantages et inconvec soin les

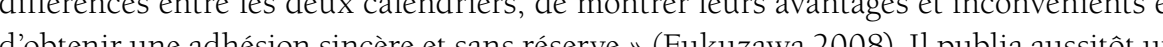
d'obtenir ne a

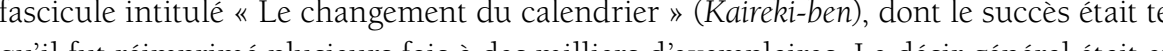

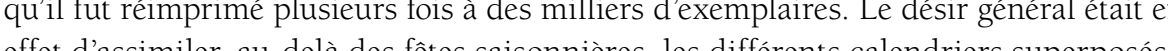
effet d assimiler, au-delà des fetes saisonnières, les difterents calendriers superposés système chinois d'organisation du temps par règne imperial - encore en application y compris pour l'édition d'ouvrages - ; système calendaire bouddhique, qui domin l'ensemble des rites funéraires ; traditions shinto liées à la fois au protocole impérial et aux grandes activités agricoles; calendrier phénologique enfin, qui associe certaines activités à la végétation naturelle et aux floraisons. Celles-ci ont lieu à des moments décalés d'une province à l'autre, en raison des conditions d'ensoleillement différentes des régions du Japon, largement étiré du nord au sud sur plus de $3000 \mathrm{~km}$ de long. Ainsi pour suivre l'éclosion des fleurs de cerisier du sud au nord du Japon, il ne faut pas moins d'un mois, ce qui rend tout à fait évidentes les différences de conditions météorologiques qui détermineront par exemple les grands travaux agricoles comme le repiquage du riz. Dans Symbolique du vêtement japonais (1946), Leroi-Gourhan montre l'importance donnée à la succession du temps et aux signes utilisés dans l'organisation matérielle pour marquer les âges successifs et les quatre saisons annuelles, ainsi que pour les différencier « dans le cycle de la vie de l'univers ». Car «à chaque âge le vêtement marque des étapes . Cest lì que réside, nous dit Leroi-Gourhan, "le plus poétique des aspects

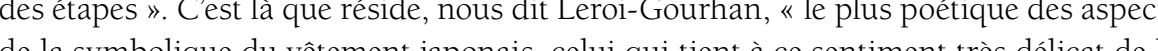

Cette interrogation japonaise sur la place de l'homme dans l'univers s'accompagne d'une préoccupation profonde sur le devenir et la métamorphose des êtres et des choses

Nul peuple na plus précise la notion du courant qui entraîne les choses vers leur

fin. Tout ce qu'il possède est empreint de fragilité et d'instabilité, tout s'use très vite,

écrit-il encore dans cet article ; et il reviendra beaucoup plus tard (Leroi-Gourhan 1984) sur cette vision japonaise du viellissement de lunivers vivant, a propos de la taphonomie, branche nouvelle de la zoologie, qui a pour but de « décrire l'évolution des organismes ou des structures vivantes depuis leur mort jusquà la dissipation de leurs vestiges ». Il en trouvera une illustration très originale dans la légende de la célèbre courtisane représentée dans toute sa beauté au début d'un rouleau qui la montre peu à peu malade, puis morte ;

[...] ici commence le cycle taphonomique qui se prolonge par la réduction de sa

tombe à quelques cailloux dispersés et de son corps à quelques fragments osseux

dévorés par les chiens sous la pluie [...]

Dans ce fameux rouleau, maintes fois reproduit, maintes fois cité, la légende veut voir la poétesse Ono no Komachi, parangon de beauté classique, sous sa splendeur éphémère (Pigeot 2003) La profonde tendance à s'attendrir sur le caractère éphémère de toute chose, et à s'émouvoir du temps qui passe, n’est en rien estompée par l'adoption du calendrier occidental au xixe siècle. Les formes calendériques peuvent diverger, ou même se contredire, la construction du temps, et la perception du temps vécu demeurent.

\section{Légende du calendrier}

Galendrier de 1989 reprenant, dans le système calendarie moderne, les noms de mois anciens, rendus en sinogrammes : mutsuki pour Janvier, kisaraggi-fevirier, yayol-mars, uzuki-avvil, satsuki-

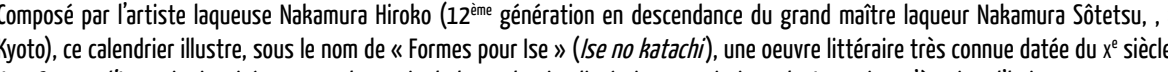

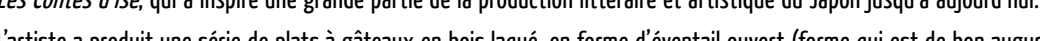

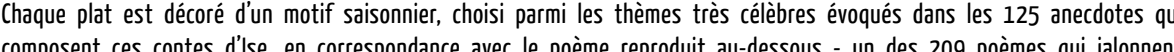
l'ensemble de cet ouvrage classique.

\section{RÉFÉRENCES}

Fukuzawa, Y. 2008 Le Changement de calendrier. In Plaidoyer pour la modernité, Introduction aux cuures completes (trad. fr. M. Saucier). Paris : Éditions du CNRS, 107-111.

Leroi-Gourhan A. 1946 Symbolique du vêtement japonais. Rythmes du monde 4 : 31-40.

- 1965 Le Geste ch la parole. Tome 2 La Memoire et les rythmes. Paris : Albin-Miche-

- 1984 L'Esprit de la tapho

Pigeot, J. 2003 Femmes galantes, femmes artistes dans le Japon ancien. Paris : Gallimard.

\section{Photo d'ouverture :}

Une page du nouvean calendrier de 1872. Ce dernier, qui est le premier du genre, indique aussi, en bas, la correspondance avec l'ancien calendrier 